\title{
Laparoscopic management of a spontaneous 12th week heterotopic tubal pregnancy: a case report
}

Published online: 23 September 2005

(C) Springer-Verlag Berlin / Heidelberg 2005

\begin{abstract}
Here we present a spontaneously-developed case involving 12 th week heterotopic tubal pregnancy. In the preoperative period, the diagnosis was confirmed both by ultrasonographic examination and magnetic resonance imaging (MRI). The patient was treated successfully using a laparoscopic salpingectomy technique without jeopardizing the intrauterine pregnancy.
\end{abstract}

Keywords Heterotopic pregnancy · Laparoscopy • Salpingectomy $\cdot$ Pregnancy

\section{Introduction}

Heterotopic pregnancy, defined as the simultaneous development of an extrauterine along with an intrauterine pregnancy, is a very rare event, even in the context of ectopic pregnancy. The incidence of heterotopic pregnancy varies between $1 / 30,000$ and $1 / 40,000$, but the prevalence of heterotopic pregnancies has been reported to rise to as high as $1-2 \%$ due to the use of assisted reproductive technology and ovulation-inducing agents $[1,2]$. Although heterotopic pregnancy is usually managed through salpingectomy performed via laparotomy, there are also reports of heterotopic pregnancies treated by laparoscopy in the literature. In fact, laparoscopy can be used safely in hemodynamically-stable patients.

In this report we present a case of spontaneously developed 12th week heterotopic tubal pregnancy that was successfully treated by salpingectomy by laparoscopy.

S. E. Akhan $(\bowtie) \cdot$ I. Çitil $\cdot$ S. Topuz $\cdot$ C. Iyibozkurt

Department of Obstetrics and Gynecology,

Istanbul University Istanbul Medical School, Çapa, Topkapı,

Istanbul, 34290, Turkey

E-mail: akhan93@hotmail.com

Tel.: + 90-212-6351150

Fax: $+90-212-6352675$

\section{Case report}

A 28-year-old gravida 1, parity 0 woman was admitted to the emergency department for acute abdomenal pain. She had experienced a sharp and generalized abdominal pain of sudden onset. On physical examination, she was found to have marked left adnexal tenderness with mild guarding and rebound. There was neither vaginal bleeding nor abnormal vaginal discharge on vaginal examination, and the cervical os was not dilated. Her blood pressure and pulse rate was $90 / 60 \mathrm{mmHg}$ and 106 beats per minute, respectively. Laboratory investigations done at this stage were: hemoglobin: $8 \mathrm{~g} / \mathrm{dl}$, WBC: 5270 , platelet 172000 , INR: 1.1. Her past medical history was unremarkable and she had no history of infertility. On ultrasonography, a healthy intrauterine $12+3$ week pregnancy with $53 \mathrm{~mm}$ CRL and an extrauterine $12+1$ week pregnancy with $48 \mathrm{~mm}$ CRL on the left side of the uterus were detected. Both fetuses had fetal cardiac activity. The diagnosis of heterotopic tubal pregnancy was considered but since the exact localization of the ectopic focus was not clear, a cornual or a possible rudimentary horn pregnancy was also included in the differential diagnosis. Finally, magnetic resonance imaging (MRI) confirmed the diagnosis of left heterotopic tubal pregnancy (Fig. 1). During the preoperative evaluation period, the patient was hemodynamically stable.

During laparoscopy, $500 \mathrm{~cm}^{3}$ of hemoperitoneum was encountered. The uterus was 12 weeks in size and an $8 \mathrm{~cm}$ ectopic pregnancy mass originated from the left ampullary region (Fig. 2). Salpingectomy was performed by successive electrocoagulation and cutting of the mesosalpinx. Salpingotomy was not considered due to both the size of the ectopic mass and the risk of excessive bleeding from the overdilated fallopian tube. Even during salpingectomy, laparoscopically manipulating adnexial mass without tearing the tubal wall was the main technical problem. The fetus and the remaining conceptus were removed through a small suprapubic incision. 


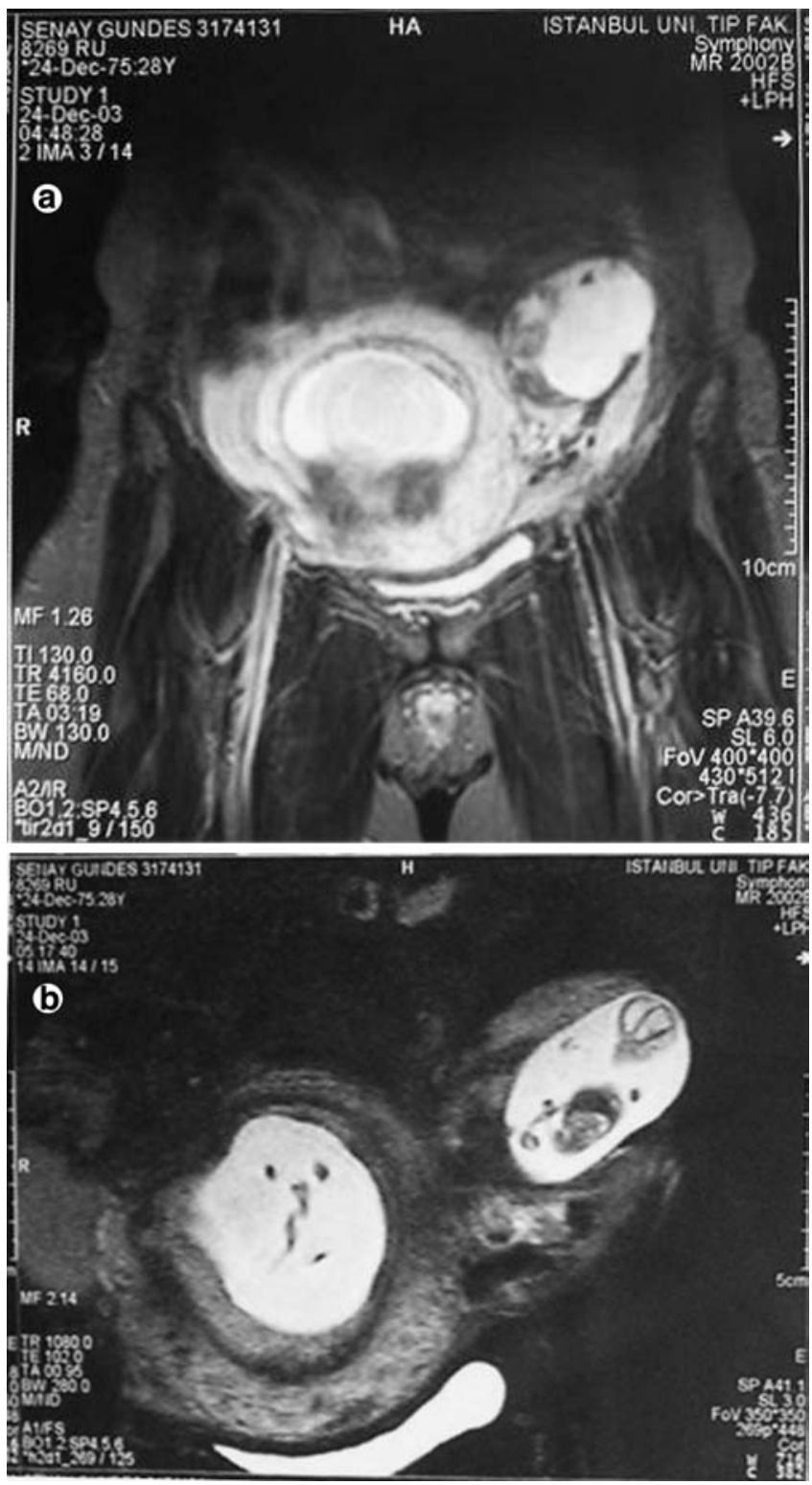

Fig. 1 An MRI performed before surgery: intrauterine and tubal pregnancy seen in axial plane (a), in coronal plane (b)

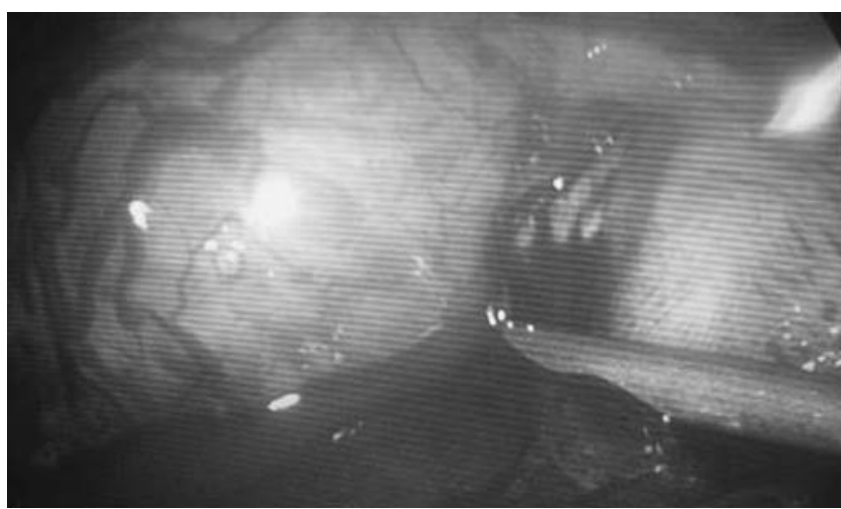

Fig. 2 An $8 \mathrm{~cm}$ tubal pregnancy mass originated from the left ampullar region
Total operation time was around $45 \mathrm{~min}$. The postoperative period was unremarkable. The patient was discharged on the third postoperative day without any complications and the ongoing pregnancy reached term uneventfully. She was delivered at the 39th week of gestation by caesarean section. The baby, measuring $49 \mathrm{~cm}$ and weighing $3200 \mathrm{~g}$, was born with Apgar scores of nine and ten at first and fifth minutes, respectively. No complications were observed until the sixth month of infancy.

\section{Discussion}

Abdominal pain, an adnexal mass, peritoneal irritation and enlarged uterus together constitute the major clinical features associated with a heterotopic pregnancy. Additional diagnostic findings during an early heterotopic pregnancy include the presence of two corpora lutea found at the time of laparotomy or laparoscopy, hemoperitoneum, acute abdominal pain after the termination of an intrauterine pregnancy and the persistence of an enlarged uterus with amenorrhea after excision of an ectopic pregnancy. The diagnosis was evident in our case, since there were two fetuses, one extrauterine, seen during ultrasonographic examination. With the increased use of assisted reproductive techniques, the incidence of this will probably increase, so one always must suspect this diagnosis in order to properly treat these patients.

Although both laparotomy and laparoscopy are appropriate procedures in the management of heterotopic pregnancies, the overall paucity of experience with laparoscopy has caused many surgeons to manage these challenging cases with laparotomy. Laparoscopic management seems to be an ideal method in properly selected patients. In the literature there is only one case, reported by Pisarska [3], in which the extrauterine component was abdominal and of six weeks gestation. It is reported that, with early intervention, up to $70 \%$ of intrauterine gestations have been salvaged regardless of the type of surgery utilized [4]. In our case, laparoscopy turned out to be an adequate treatment modality for a heterotopic pregnancy of 12 weeks of gestation.

Laparoscopy is emerging as an alternative to laparotomy for definitive diagnosis and treatment of surgical conditions arising in pregnancy. General principles of laparoscopic surgery are also applicable to pregnant women. Operative field exposure is excellent and not yet compromised by the enlarged uterus during early pregnancy. Carbon dioxide pneumatic distention is the dominant modality in current practice. Unresolved controversy surrounds the use of carbon dioxide as a peritoneal distension medium and this centers on the immediate and delayed effects of vascular compression and transperitoneal carbon dioxide absorption; but prospective studies are lacking on this issue. A growing body of literature on pneumoperitoneum suggests that carbon dioxide does not cause a teratogenic or an otherwise detrimental effect on newborns. However, a 
study of carbon dioxide pneumoperitoneum in pregnant ewes demonstrated no adverse effects from increased pressure alone, but increased incidence of fetal hypercarbia, acidosis, increased fetal arterial pressure and possible tachycardia associated with the use of carbon dioxide [5]. We therefore need further studies on the safety of laparoscopy for the fetus and newborns. The only comparative analysis evaluating the effect of laparoscopy and laparotomy in pregnancy on fetal outcome demonstrated no differences with regard to birth, gestational duration, growth restriction, infant survival and fetal malformation [6]. Furthermore, little information is available on the growth and development of infants who survive the neonatal period, but it is suggested that the incidence of congenital malformations and mental retardation may be increased due to hypotension from the extrauterine pregnancy [7]. In our case, the infant was neurologically and physically normal until six months of age.

\section{References}

1. Tal J, Haddad S, Gordon N, Timor-Trisch I (1996) Heterotopic pregnancy after ovulation induction and assisted reproductive technologies: a literature review from 1971 to 1993. Fertil Steril 66:1

2. Tummon IS, Whitmore NA, Daniel SAJ et al (1994) Transferring more embryos increases risk of heterotopic pregnancy. Fertil Steril 61:1065

3. Pisarska M, Casson RP, Moise Kenneth et al (1998) Heterotopic abdominal pregnancy treated at laparoscopy. Fertil Steril 70:159

4. Rojansky N, Schenker JG (1996) Heterotopic pregnancy and assisted reproduction: an update. J Assist Reprod Genet 13:594

5. Hunter JG, Swanstrom L, Thornburg K (1995) Carbon dioxide pneumoperitoneum induces fetal acidosis in pregnant ewe. Surg Endose 9:272

6. Reedy MB, Kalen B, Kuehl TJ (1997) Laparoscopy during pregnancy: a study of five fetal outcome parameters with use of Swedish health registry. Am J Obstet Gynecol 177:673

7. Reece EA, Petrie RH, Sirmans MF et al (1983) Combined intrauterine and extrauterine gestations: a review. Am J Obstet Gynecol 146:323 